\title{
Relación entre el gasto en investigación y desarrollo con la producción científica en el Perú
}

\author{
Relationship between expenditure in research and development and scientific production
}

in Peru

\author{
Ricardo Robles-Alfaro ${ }^{1,2, a}$, Fernando Vela-Alfaro ${ }^{1,2, a}$, Oscar Huapaya-Huertas ${ }^{3, b}$, \\ Horacio Chacón-Torrico ${ }^{3, b}$ \\ 'Facultad de Medicina Humana, Universidad Cientifica del Sur, Lima, Perú. \\ ${ }^{2}$ Sociedad Cientifica de Estudiantes de Medicina de la Universidad Cientifica del Sur (SCIEM - UCSUR), Lima, Perú. \\ ${ }^{3}$ Centro de Investigación ANASTOMOSIS, Lima, Perú. \\ ${ }^{a}$ Estudiante de Medicina; ${ }^{\mathrm{b}}$ Médico cirujano.
}

\section{An Fac med. 2015;76(4):469-70 / http://dx.doi.org/10.15381/anales.v76i4.11423}

\section{SR. EDITOR:}

En el mundo, la producción científica tiene un incremento anual estimado de $3 \%$ y un volumen de publicaciones que se duplica cada 24 años ${ }^{(1)}$. Sin embargo, este crecimiento no es homogéneo entre todas las regiones. En América Latina la producción científica publicada entre 1996 y 2014 representó el 3,39\% de la producción mundial; y, a su vez, el Perú contribuyó en esos años con solo $1,04 \%$ de dicha producción en la región ${ }^{(2)}$.

Se han descrito múltiples causas para la baja producción científica pe- ruana ${ }^{(3,4)}$, pero pocos la han asociado con el producto bruto interno (PBI), en particular, con el casto interno bruto en investigación y desarrollo (GIBID). Es por esto que se recogió los valores en cuanto a la producción científica de los tres países latinoamericanos con mayor producción (Brasil, México y Argentina), y se comparó con los valores del Perú. La información fue recolectada de la base de datos SCImago Journal $\mathbb{E}$ Country Rank (SJR) ${ }^{(2)}$, que incluye solo publicaciones en Scopus. A su vez, se determinó el GIBID de estos países, a partir del PBI y el porcentaje del PBI gastado en investigación y desarrollo
(GIBID\%) obtenidos del Banco Mundial ${ }^{(5)}$. Los datos del GIBID\% referentes al Perú provienen de la información brindada por el Consejo Nacional de Ciencia, Tecnología e Innovación Tecnológica (CONCYTEC) desde 1997 hasta el $2004^{(5)}$.

En la gráfica 1, se percibe una correlación progresiva entre la producción científica y el GIBID; dicho de otra manera, los países con mayor inversión en investigación y desarrollo presentan mayores niveles de producción científica. Esto puede reflejar una relación significativa entre estas variables, aunque no ha sido aún evaluada estadística-
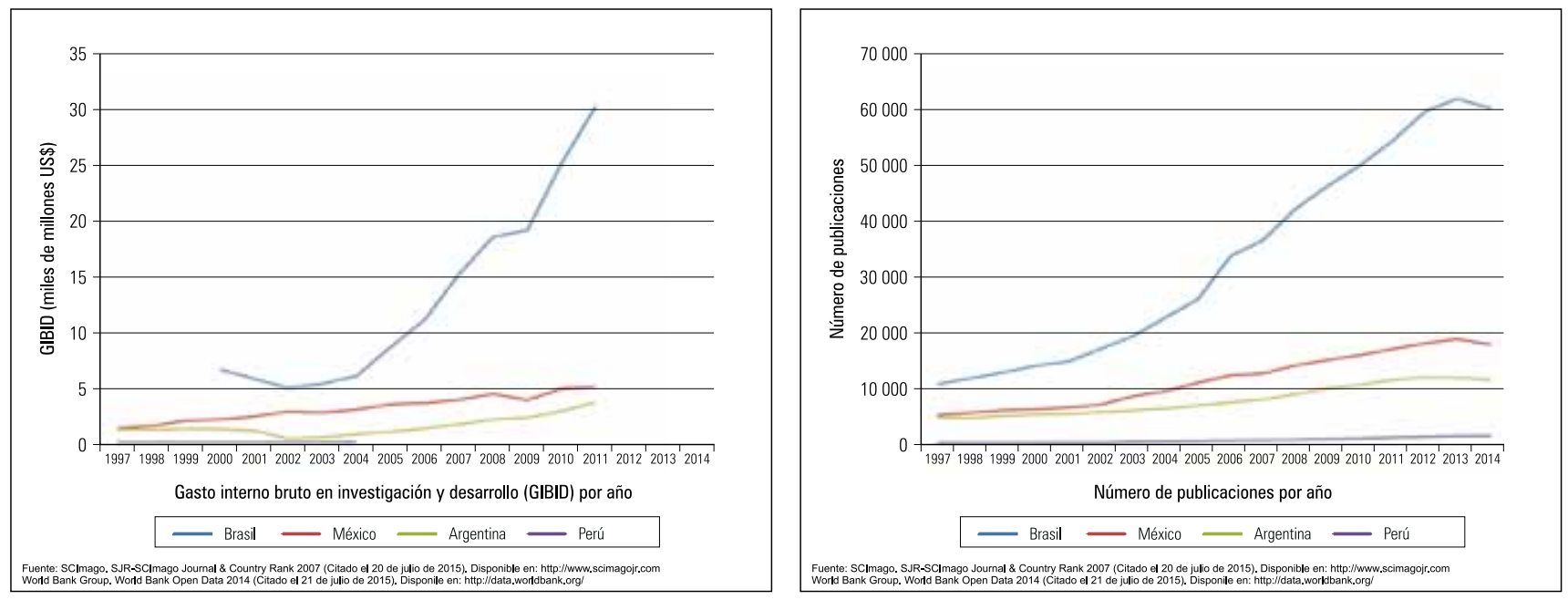

Figura 1. Correlación entre el número de publicaciones y GIBID de los países latinoamericanos con mayor producción y el Perú, $1997-2013$. 
mente; de ser así, dentro de las causas de baja productividad, la escasa inversión en investigación sería el único factor con la particularidad de ser medible. Es necesario recalcar que el CONCYTEC no ha revelado los datos del GIBID\% desde el 2004, por lo que, la valoración realizada se ve cubierta por un amplio margen de desconocimiento en relación con los datos estadísticos actuales, probablemente porque no existe un sistema integrado y consolidado de información sobre el Sistema Nacional de Ciencia, Tecnología e Innovación Tecnológica (SINACYT) ${ }^{(6)}$. Asimismo, cabe señalar la lamentable y preocupante baja productividad que el Perú presenta, en comparación con los otros países, siendo esta proporcional al bajo nivel de inversión en investigación. Dicho lo anterior, el CONCYTEC pretende fortalecer y renovar la eficiencia del Sistema Nacional de Innovación con una serie de metas, dentro de las cuales se incluye mejorar el GIBID para los próximos años ${ }^{(6)}$. Mediante estas medidas, se espera que las instituciones peruanas dedicadas a la investigación y desarrollo sean competentes a nivel global, permitiendo mejores oportunidades laborales, económicas y sociales para las futuras generaciones.

\section{REFERENCIAS BIBLIOGRÁFICAS}

1. Bornmann L, Mutz R. Growth rates of modern science: a bibliometric analysis based on the number of publications and cited references. J Assoc Inform Science Technol. 2015. DOI: 10.1002/asi.23329.

2. SCImago. SJR - SCImago Journal \& Country Rank 2007 [Citado el 20 de julio del 2015 ]. Disponible en:: http://www.scimagojr.com.

3. Huamaní C, Chávez-Solis $\mathrm{P}$, Mayta-Tristán $\mathrm{P}$. Aporte estudiantil en la publicación de artículos científicos en revistas médicas indizadas en ScieloPerú, 1997-2005. An Fac med. 2008;69(1):42-5.

4. Pamo Reyna OG. Estado actual de las publicaciones periódicas científicas médicas del Perú. Rev Med Herediana. 2005;16:65-73.

5. World Bank Group. World Bank Open Data 2014 [Citado el 21 de julio del 2015]. Disponible en: http://data.worldbank.org/.

6. Orjeda-Fernández G. Crear para crecer. Estrategia Nacional para el Desarrollo de la Ciencia, Tecnología e Innovación. 2014.

Carta al Editor recibida el 28 de julio de 2015.

Financiamiento: Autofinanciamiento

Conflictos de interés.

Los autores declaran no tener conflictos de interés.

Correspondencia:

Ricardo Alessandro Robles Alfaro

Dirección: Jr. Daniel Hernández 1329 - Pueblo Libre

Teléfono: 971128208

Correo electrónico: r robles_a@live.com 